\title{
Stability of control systems under aperiodic sampling and input saturation with clock-dependent Lyapunov functions *
}

\author{
Arthur Scolari Fagundes* João Manoel Gomes da Silva Jr. * \\ Marc Jungers** \\ * Programa de Pós graduação em Engenharia Elétrica, \\ Universidade Federal do Rio Grande do Sul (UFRGS). \\ (e-mails:arthur.fagundes@ufrgs.br; jmgomes@ufrgs.br) \\ ** Université de Lorraine, CNRS, CRAN, F-54000 Nancy, France. \\ (e-mail: marc.jungers@univ-lorraine.fr)
}

\begin{abstract}
In this work, a new method of stability analysis of sampled-data control systems with input saturation is proposed. With the use of a class of time-dependent Lyapunov functions, the continuous and discrete dynamics of the system are taken into account to assess the stability of the closed-loop system when aperiodic sampling is considered. Considering a particular quadratic form of this class of Lyapunov functions, new conditions to estimate the region of attraction of such nonlinear systems are derived.
\end{abstract}

Keywords: Aperiodic sampling; Clock-dependent Lyapunov; Control saturation; Sampled-data system;

\section{INTRODUCTION}

Much attention has been devoted to the stability analysis of sampled-data control systems in the past decades (Hespanha et al., 2007). A sampled-data system consists in a plant evolving in continuous-time and a controller setting inputs to that plant in discrete-time (Seuret and Gomes da Silva Jr., 2012). On one side, in the last years of the previous century an intensive research on sampled-data systems under periodic sampling has been developed (see Aström and Wittenmark (1984) and Chen and Francis (1994)). On the other side, the rising importance of the field of networked control systems has brought new challenges to the application of control theory (P. Antsakils, 2007), one very important being the feature of aperiodic sampling (i.e., sampling with variable sampling intervals) imposed by the network. For a sensor to transmit a continuoustime signal over a network, the signal must be sampled, converted to a digital format, transmitted, and finally the data must be converted again to the analog format to be received at the controller side. The total delay between sampling and eventual reception is subject to significant variations due to network conditions such as congestion and eventual packet drop-outs (Hespanha et al., 2007), and motivates the study of aperiodic sampled-data systems.

The study of aperiodic sampled-data systems is quite underdeveloped compared to the periodic counterpart (Hetel et al., 2016), but has been receiving attention recently in the context of control theory. One way to represent it is

\footnotetext{
* This study was financed in part by the Coordenação de Aperfeiçoamento de Pessoal de Nível Superior - (CAPES/Brazil) Finance Code 001, by the CNPq/Brazil (grants PQ:305979/2015-9 and Universal: 422992/2016-0), and ANR/France (project HANDY, number ANR18-CE40-0010).
}

by an impulsive system model. This system framework emerges as a generalization of the discrete-time model which considers the inter-sampling system behaviour using signal lifting (see Hetel et al. (2016) and references therein). For instance, in Geromel and Souza (2005) an impulsive system framework is used to provide conditions for the stabilization of sampled-data linear systems, aiming at optimizing $\mathrm{H}_{2}$ and $\mathrm{H}_{\infty}$ criteria. More recently, in Geromel and Souza (2005), the synthesis of dynamic output feedback using differential matrix inequalities is addressed. It should be highlighted that the above references do not consider the possibility of actuator saturation.

In the present paper, the almost ubiquitous feature of input saturation is taken into account. Numerous works consider the problems of stability analysis of systems with input saturation (see Hu and Lin (2001) and Tarbouriech et al. (2011)). Many of these approaches have been proposed considering either continuous or discrete-time systems. Considering the periodic sampling case, the problem of assessing stability of a samped-data system with dynamic output feedback control law has been addressed in Dai et al. (2009). Aperiodic sampling in the presence of control saturation is addressed in Seuret and Gomes da Silva Jr. (2012) and Gomes da Silva Jr. et al. (2016) considering a looped-functional approach. In Fiacchini and Gomes da Silva Jr. (2018) this problem is addressed considering an impulsive system framework and the discrete-time dynamics of the closed-loop system cast as a difference inclusion obtained from a partition of the intersampling interval.

In the present paper we are interested in the problem of stability analysis of an aperiodic sampled-data system subject to input saturation. From an impulsive system rep- 
resentation of the closed-loop system it is shown that, by satisfying stability conditions based on a clock-dependent Lyapunov function, a monotonically decreasing quadratic form of this class of function ensures the local stability of the system equilibrium point. Moreover, the conditions can be cast in LMI-based optimization problems to generate piecewise quadratic estimates of the region of attraction of the origin.

The content of this paper is organized as follows: In section 2, the impulsive representation of the sampled-data controlled system is presented. Section 3 regards Lyapunov stability theorem and its statement for the clockdependent class of functions. In section 4 , the saturation effect is included in the modeling, and stability conditions dealing with this nonlinearity are derived. In section 5 an optimization problem to generate estimates of the region of attraction of the origin is stated, and a numerical example is exposed in section 6 .

Notation: $\mathbb{N}$ is the set of natural numbers, $\mathbb{R}$ is the set of real numbers, and $\mathbb{R}^{+}$is the set of positive real numbers. For a function $x: \mathbb{R} \rightarrow \mathbb{R}^{n}$, we define

$$
x\left(t^{-}\right)=\lim _{\tilde{t} \rightarrow t, \tilde{t}<t} x(\tilde{t})
$$

The $i$ th element of a vector $v$ is denoted by $v_{(i)}$, the element in the $i$ th row and $j$ th column of a matrix $M$ is denoted by $M_{(i, j)}$, while $M_{(i)}$ denotes the entire row $i$ of $M$. Note that the indexation of lines and elements only occurs between parentheses - in $M_{m}, m$ is simply a subscript. $\mathbb{S}^{n}$ is the domain of symmetric matrices of order $n$, and for a symmetric matrix $S \in \mathbb{S}^{n}, S>0$ means that $S$ is positive definite. $M^{\prime}$ denotes the transpose of $M . I$ denotes a identity matrix of appropriate dimensions.

\section{CONTROL UNDER APERIODIC SAMPLING}

Consider the continuous-time plant described by the following linear time-invariant model:

$$
\dot{x}_{p}(t)=A x_{p}(t)+B u(t)
$$

where $x_{p} \in \mathbb{R}^{n_{p}}$ represents the state of the plant, $u \in \mathbb{R}^{m}$ represents its input, and $t \in \mathbb{R}^{+}$is time. Matrices $A$ and $B$ have appropriate dimensions and are constant.

It is considered that the input signal is calculated from samples, as if coming from a digital controller, and obeys the control law given by

$$
u(t)=K x_{p}\left(t_{k}\right) \quad \forall t \in\left[t_{k}, t_{k+1}\right), \quad \forall k \in \mathbb{N}
$$

with $t_{k}, k \in \mathbb{N}$, being the sampling instants. $u(t)$ is supposed to be kept constant between two successive sampling instants through a zero order holder.

Since we are considering an aperiodically sampled control, the time between two successive samples $\delta_{k}=t_{k+1}-t_{k}$ is not necessarily constant. In particular, we assume that

$$
0<\underline{\tau} \leq \delta_{k} \leq \bar{\tau}
$$

The bounds $\underline{\tau}$ and $\bar{\tau}$ are supposed to be imposed by constraints on the networked control implementation. As stated in the introduction, they represent network conditions that affect sampling rate e.g. a lag induced by the communication protocol.

Note that assuming $\underline{\tau}>0$ is sufficient to avoid Zeno effect. The sequence of admissible sampling intervals $\Theta$ can be formalized by the set below

$\Theta=\left\{t=t_{k} \in \mathbb{R}^{+}: t_{k+1}=t_{k}+\delta_{k}, \quad \delta_{k} \in[\underline{\tau}, \bar{\tau}], \forall k \in \mathbb{N}\right\}$

The closed loop system can thus be described by the following system.

$$
\dot{x}_{p}(t)=A x_{p}(t)+B K x_{p}\left(t_{k}\right) \quad \forall t \in\left[t_{k}, t_{k+1}\right)
$$

If we want to describe it for all $t \geq 0$ and a particular sampling sequence $\theta \in \Theta$, the following impulsive model can be considered:

$$
\left\{\begin{array}{lc}
\dot{x}(t)=A_{f} x(t) & \forall t \notin \theta ; \quad t \neq 0 \\
x(t)=J_{j} x\left(t^{-}\right) & \forall t \in \theta ; \quad t \neq 0 \\
x_{p}(0)=x_{0} & t=0 \\
u(0)=K x_{0} & t=0
\end{array}\right.
$$

where $x(t)=\left[x_{p}(t)^{\prime} u(t)^{\prime}\right]^{\prime} \in \mathbb{R}^{n}$, with $n=n_{p}+m$, is the overall system state, and $A_{f}$ and $J_{j} \in \mathbb{R}^{n \times n}$ are given as follows

$$
A_{f}=\left[\begin{array}{cc}
A & B \\
0 & 0
\end{array}\right], \quad J_{j}=\left[\begin{array}{cc}
I & 0 \\
K & 0
\end{array}\right]
$$

\section{CLOCKED-LYAPUNOV STABILITY THEOREM}

The following Theorem provides conditions to assess the stability of the hybrid system (2). (Hetel et al., 2016)

Theorem 1. Consider system (2) and denote $\tau=\tau_{k}(t)=$ $t-t_{k}, \forall t \in\left[t_{k}, t_{k+1}\right), \forall k \in \mathbb{N}$ the clock variable. Let $c_{1}$, $c_{2}$ and $c_{3}$ be positive constants, and $V$ be a function such that

$$
c_{1}\|x\|^{2} \leq V(x, \tau) \leq c_{2}\|x\|^{2}
$$

for all $x \in \mathbb{R}^{n}, \tau \in[0, \bar{\tau}]$. Suppose that for any impulse sequence $\theta \in \Theta$ the corresponding solution $x(t)$ to $(2)$ satisfies:

$$
\frac{d V\left(x(t), \tau_{k}(t)\right)}{d t}<-c_{3}\|x(t)\|^{2} \quad \forall t \in\left[t_{k}, t_{k+1}\right), \quad \forall k \in \mathbb{N}
$$

and

$$
V\left(x\left(t_{k}\right), \tau_{k}\left(t_{k}\right)\right)<V\left(x\left(t_{k}^{-}\right), \tau_{k}\left(t_{k}^{-}\right)\right) \quad \forall k \in \mathbb{N}
$$

then, the equilibrium point $x=0$ of system (2) is globally exponentially stable.

Note that for $t \in\left[t_{k}, t_{k+1}\right)$, i.e., $t \notin \theta \cup\{0\}$, it follows from (1) that $\tau_{k}(t) \in[0, \bar{\tau})$, and also that $\tau_{k}\left(t_{k}\right)=0$. Moreover, one has 


$$
\dot{\tau}_{k}(t)=\frac{d \tau_{k}(t)}{d t}=1 \quad \forall t \notin \theta \cup\{0\}
$$

Based on these facts the following corollary can be stated.

Corollary 1. If there exists a differentiable function $\hat{V}(x, \tau)=x(t)^{\prime} P(\tau) x(t), \quad P:[0, \bar{\tau}] \rightarrow \mathbb{S}^{n}, P(\tau)>$ $0, \quad \forall \tau \in[0, \bar{\tau})$, such that

$$
A_{f}^{\prime} P(\tau)+P(\tau) A_{f}+\dot{P}(\tau)<0, \quad \forall \tau \in[0, \bar{\tau})
$$

and

$$
J_{j}^{\prime} P(0) J_{j}-P(\tau)<0, \quad \forall \tau \in[\underline{\tau}, \bar{\tau}]
$$

are satisfied, then the equilibrium point $x=0$ of system (2) is globally exponentially stable.

Proof. From (5), $\dot{\tau}=1$ for $t \notin \theta \cup\{0\}$. Hence, inequality (3) in Theorem 1 is given by

$$
\begin{aligned}
& \frac{d \hat{V}\left(x(t), \tau_{k}(t)\right)}{d t}=\frac{d \hat{V}}{d x} \dot{x}+\frac{d \hat{V}}{d \tau} \dot{\tau} \\
= & \dot{x}^{\prime} P\left(\tau_{k}(t)\right) x+x^{\prime} P\left(\tau_{k}(t)\right) \dot{x}+x^{\prime} \dot{P}\left(\tau_{k}(t)\right) x<0 .
\end{aligned}
$$

As $\tau_{k}(t) \in[0, \bar{\tau}]$, and taking into account (2)-a, in order to satisfy (8) it suffices that

$$
A_{f}^{\prime} P(\tau)+P(\tau) A_{f}+\dot{P}(\tau)<0 \quad \forall \tau \in[0, \bar{\tau})
$$

which implies that exists $c_{4}=\lambda_{\max }\left(A_{j}^{\prime} P(\tau)+P(\tau) A_{j}+\right.$ $\dot{P}(\tau))$, for $\tau \in[0, \bar{\tau}]$, where $\lambda_{\max }(\cdot)$ is the maximum eigenvalue of a matrix.

On the other hand, for $t \in \theta$, we have $\tau_{k}(t)=0$ and thus, from (2)-b, inequality (4) in Theorem 1 is satisfied if

$$
J_{j}^{\prime} P(0) J_{j}-P(\tau)<0 \quad \forall \tau \in[\underline{\tau}, \bar{\tau}]
$$

which concludes the proof.

\section{STABILITY ANALYSIS WITH SATURATION}

We consider now the case in which the control signal is constrained in amplitude by means of a saturating actuator. The actual plant input in this case is given as follows

$$
u(t)=\operatorname{sat}\left(K\left(x_{p}\left(t_{k}\right)\right)\right)
$$

where $\operatorname{sat}(\cdot): \mathbb{R}^{m} \rightarrow \mathbb{R}^{m}$ is a vector valued saturation function, i.e.

$$
\operatorname{sat}(v)_{(i)}=\operatorname{sign}\left(v_{(i)}\right) \min \left(\left|v_{(i)}\right|, 1\right) \quad \forall i=1, \cdots, m
$$

With saturation in the controller output, the closed-loop system (2) must be rewritten as follows:

$$
\left\{\begin{array}{lc}
\dot{x}(t)=A_{f} x(t) & \forall t \notin \theta ; \quad t \neq 0 \\
x(t)=A_{j} x\left(t^{-}\right)+B_{j} \operatorname{sat}\left(K_{j} x\left(t^{-}\right)\right) & \begin{array}{l}
t \in \theta ; \\
t \neq 0
\end{array} \\
x_{p}(0)=x_{0} & t=0 \\
u(0)=\operatorname{sat}\left(K x_{0}\right) & t=0
\end{array}\right.
$$

where $A_{f}, A_{j} \in \mathbb{R}^{n \times n}, B_{j} \in \mathbb{R}^{n \times m}$ and $K_{j} \in \mathbb{R}^{m \times n}$ are given as follows

$$
A_{f}=\left[\begin{array}{cc}
A & B \\
0 & 0
\end{array}\right], \quad A_{j}=\left[\begin{array}{ll}
I & 0 \\
0 & 0
\end{array}\right], \quad B_{j}=\left[\begin{array}{l}
0 \\
I
\end{array}\right], \quad K_{j}=\left[\begin{array}{ll}
K & 0
\end{array}\right]
$$

Although the origin of a linear closed-loop system is supposed to be either globally asymptotically stable or not stable in the absence of input constraints, this is not the case when saturation is present. In the case of control systems with input saturation, the stability of an equilibrium point is not necessarily global. In this case it is important to characterize the region of attraction of the origin and its estimates. The approach used here to estimate the region of attraction of the origin can be found in Tarbouriech et al. (2011).

Aiming to express stability conditions specifically for system (10), the quadratic function $\hat{V}$ given in Corollary 1 will be used to rewrite conditions (3) and (4) of Theorem 1 as matrix inequalities and therefore to formulate a convex optimization problem to maximize the estimated region of attraction of the system. With that in mind, the first step concerns the treatment of the saturation term of system (10). The idea in this case is to use a deadzone nonlinearity and a corresponding generalized sector condition as a mean of relaxing the problem. A deadzone function can be defined from the saturation function given in (9) as follows

$$
\mathrm{dz}(v)=\operatorname{sat}(v)-v
$$

that is

$$
\mathrm{dz}(v)_{(i)}=\operatorname{sign}\left(v_{(i)}\right)\left(1-\max \left(\left|v_{(i)}\right|, 1\right)\right)
$$

Regarding the deadzone nonlinearity, the following lemma can be stated:

Lemma 1. (Tarbouriech et al., 2011) Consider $K_{j}, G_{j} \in$ $\mathbb{R}^{m \times n}$ and define the set

$$
\mathcal{S}=\left\{x \in \mathbb{R}^{n} ;\left|\left(K_{j(i)}-G_{j(i)}\right) x\right| \leq 1, i=1, \cdots, m\right\}
$$

If $x \in \mathcal{S}$, then the deadzone nonlinearity $d z\left(K_{j} x\right)$ satisfies the following inequality

$$
\mathrm{dz}\left(K_{j} x\right)^{\prime} T\left(\mathrm{dz}\left(K_{j} x\right)+G_{j} x\right) \leq 0
$$

for any diagonal positive definite matrix $T \in \mathbb{R}^{m \times m}$.

In order to apply the property stated in Lemma 1 in the stability analysis, saturation function in (10)-b must be substituted by the deadzone function. Note that:

$$
\operatorname{sat}\left(K_{j}\left(x\left(t_{k}^{-}\right)\right)=K_{j}\left(x\left(t_{k}^{-}\right)\right)+\mathrm{dz}\left(K_{j}\left(x\left(t_{k}^{-}\right)\right)\right)\right.
$$

The substitution of (14) in (10)-b results in

$$
\begin{aligned}
& x(t)= \mathbb{A}_{j} x\left(t^{-}\right)+B_{j} \mathrm{dz}\left(K_{j}\left(x\left(t_{k}^{-}\right)\right) \forall t \in \theta\right. \\
& \mathbb{A}_{j}=A_{j}+B_{j} K_{j}
\end{aligned}
$$

Next we aim to provide a condition to ensure the local exponential stability of the origin of system (10) along with an estimate of its region of attraction.

For this, we will consider the application of Theorem 1 to system (10) with a quadratic Lyapunov candidate function that depends affinely on $\tau$, defined as follows:

$$
\begin{aligned}
\hat{V}\left(x(t), \tau_{k}(t)\right) & = \\
x^{\prime}(t) P\left(\tau_{k}(t)\right) x(t) & =x(t)^{\prime}\left(P_{1}+\tau_{k}(t) P_{2}\right) x(t)
\end{aligned}
$$

In order to state the main result, the following instrumental result is recalled 
Lemma 2. (Finsler, 1937) For $y \in \mathbb{R}^{2 n+m}, \Lambda=\Lambda^{\prime} \in$ $\mathbb{R}^{2 n+m \times 2 n+m}$ and $\Gamma \in \mathbb{R}^{n \times 2 n+m}$ with $\operatorname{rank}(\Gamma)<n$. The following statements are equivalent:

(1) $y^{\prime} \Lambda y<0 \quad \forall y \neq 0: \Gamma y=0$

(2) $\exists N \in \mathbb{R}^{2 n+m \times n}: \Lambda+N \Gamma+\Gamma^{\prime} N^{\prime}<0$

Based on Lemmas 1 and 2, and the conditions in Theorem 1 with the quadratic function (16), we can now state a testable condition for the stability analysis of system (10).

Theorem 2. If there exist matrices $P_{1} \in \mathbb{S}^{n}, P_{2} \in \mathbb{S}^{n}$, $G_{j} \in \mathbb{R}^{m \times n}, T \in \mathbb{R}^{m \times m}$ and $N \in \mathbb{R}^{2 n+m \times n}$ that satisfy the following matrix inequalities:

$$
\begin{aligned}
& \Lambda_{1}+N \Gamma+\Gamma^{\prime} N^{\prime}<0 \\
& \Lambda_{2}+N \Gamma+\Gamma^{\prime} N^{\prime}<0 \\
& A_{f}^{\prime} P_{1}+P_{1} A_{f}+P_{2}<0 \\
& A_{f}^{\prime}\left(P_{1}+\bar{\tau} P_{2}\right)+\left(P_{1}+\bar{\tau} P_{2}\right) A_{f}+P_{2}<0 \\
& P_{1}>0 \\
& P_{1}+\bar{\tau} P_{2}>0 \\
& {\left[\begin{array}{cc}
\left(P_{1}+\bar{\tau} P_{2}\right) & \left(K_{j(i)}-G_{j(i)}\right)^{\prime} \\
\star & 1
\end{array}\right] \geq 0} \\
& {\left[\begin{array}{cc}
\left(P_{1}+\underline{\tau} P_{2}\right) & \left(K_{j(i)}-G_{j(i)}\right)^{\prime} \\
\star & 1
\end{array}\right] \geq 0, \quad i=1, \cdots, m}
\end{aligned}
$$

with

$$
\begin{aligned}
& \Lambda_{1}=\left[\begin{array}{ccc}
P_{1} & 0 & 0 \\
\star & -P_{1}-\underline{\tau} P_{2} & -G_{j}^{\prime} T \\
\star & \star & -2 T
\end{array}\right] \\
& \Lambda_{2}=\left[\begin{array}{ccc}
P_{1} & 0 & 0 \\
\star & -P_{1}-\bar{\tau} P_{2} & -G_{j}^{\prime} T \\
\star & \star & -2 T
\end{array}\right] \\
& \Gamma=\left[\begin{array}{lll}
-I & \mathbb{A}_{j} & B_{j}
\end{array}\right]
\end{aligned}
$$

then, for all initial conditions of system (10) in the ellipsoid $\mathcal{E}(P(0))=\left\{x \in \mathbb{R}^{n}: x^{\prime} P_{1} x \leq 1\right\}$, the corresponding trajectories converge exponentially to the origin $\forall \theta \in \Theta$.

Proof. Consider the function $\hat{V}\left(x(t), \tau_{k}(t)\right)$ defined in (16). In order to satisfy condition (3) of Theorem 1 we must ensure that:

$$
\begin{aligned}
\dot{\hat{V}}\left(x(t), \tau_{k}(t)\right)= & x(t)^{\prime}\left[\left(P_{1}+\tau_{k}(t) P_{2}\right) A_{f}\right. \\
& \left.+A_{f}^{\prime}\left(P_{1}+\tau_{k}(t) P_{2}\right)+P_{2}\right] x(t)<0, \\
& \forall t \in\left(t_{k}, t_{k+1}\right)
\end{aligned}
$$

which is ensured if

$$
\begin{array}{r}
\left.\left(P_{1}+\tau_{k}(t) P_{2}\right) A_{f}+A_{f}^{\prime}\left(P_{1}+\tau_{k}(t) P_{2}\right)+P_{2}\right)<0 \\
\forall t \in\left(t_{k}, t_{k+1}\right)
\end{array}
$$

As $\tau_{k}(t) \in[0, \bar{\tau}), \forall t \in\left[t_{k}, t_{k+1}\right)$ and (16) has affine dependence on $\tau_{k}(t)$, a necessary and sufficient condition to satisfy (3) is given by relations (19) and (20).

Let us now consider condition (4) in Theorem 1. This condition evaluated on the system trajectories can be stated as follows:

$$
\left[\begin{array}{c}
x\left(t_{k}\right) \\
x\left(t_{k}^{-}\right)
\end{array}\right]^{\prime}\left[\begin{array}{cc}
P(0) & 0 \\
\star & -P\left(\tau_{k-1}\left(t_{k}\right)\right)
\end{array}\right]\left[\begin{array}{l}
x\left(t_{k}\right) \\
x\left(t_{k}^{-}\right)
\end{array}\right]<0
$$

for $\left[x\left(t_{k}\right)^{\prime} x\left(t_{k}^{-}\right)^{\prime} \mathrm{dz}\left(K_{j}\left(x\left(t_{k}^{-}\right)\right)\right)^{\prime}\right]^{\prime}$ such that

$$
\left[\begin{array}{lll}
-I & \mathbb{A}_{j} & B_{j}
\end{array}\right]\left[\begin{array}{c}
x\left(t_{k}\right) \\
x\left(t_{k}^{-}\right) \\
\mathrm{dz}\left(K_{j}\left(x\left(t_{k}^{-}\right)\right)\right)
\end{array}\right]=0
$$

Supposing now that $x\left(t_{k}^{-}\right) \in \mathcal{S}$, it follows from Lemma 1 that

$$
\left[\begin{array}{c}
x\left(t_{k}\right) \\
x\left(t_{k}^{-}\right) \\
\mathrm{dz}\left(K_{j}\left(x\left(t_{k}^{-}\right)\right)\right)
\end{array}\right]^{\prime}\left[\begin{array}{ccc}
0 & 0 & 0 \\
\star & 0 & -G_{j}^{\prime} T \\
\star & -2 T
\end{array}\right]\left[\begin{array}{c}
x\left(t_{k}\right) \\
x\left(t_{k}^{-}\right) \\
\mathrm{dz}\left(K_{j}\left(x\left(t_{k}^{-}\right)\right)\right)
\end{array}\right] \leq 0
$$

Hence, applying the S-procedure and Lemma 2, it follows that if

$$
\Lambda\left(\tau_{k-1}\left(t_{k}\right)\right)+N \Gamma+\Gamma^{\prime} N^{\prime}<0
$$

with

$$
\Lambda\left(\tau_{k-1}\left(t_{k}\right)\right)=\left[\begin{array}{ccc}
P(0) & 0 & 0 \\
\star & -P\left(\tau_{k-1}\left(t_{k}\right)\right) & -G_{j}^{\prime} T \\
\star & \star & -2 T
\end{array}\right]
$$

then (28) is verified.

Recalling now that $\tau_{k-1}\left(t_{k}\right) \in[\underline{\tau}, \bar{\tau})$, as condition (32) is affine in $\tau_{k-1}\left(t_{k}\right)$, a necessary and sufficient condition to verify (32) is given by matrix inequalities (17) and (18).

Suppose now that $x(0)=x\left(t_{0}\right) \in \mathcal{E}(P(0))$. From (19) and (20) it follows that $x\left(t_{1}^{-}\right) \in \mathcal{E}\left(P\left(t_{1}^{-}\right)\right)$, since

$$
x\left(t_{1}^{-}\right)^{\prime} P\left(\tau_{0}\left(t_{1}^{-}\right)\right) x\left(t_{1}^{-}\right)<x\left(t_{0}\right)^{\prime} P(0) x\left(t_{0}\right) \leq 1
$$

Now, from conditions (23) and (24), as $x\left(t_{1}^{-}\right) P\left(\tau_{0}\left(t_{1}^{-}\right)\right) x\left(t_{1}^{-}\right)<$ 1 , it follows that $x\left(t_{1}^{-}\right) \in \mathcal{S}$. On the other hand, conditions (23) and (24) ensure that $\mathcal{E}\left(P\left(\tau_{k-1}\left(t_{k}^{-}\right)\right)\right) \subset$ $\mathcal{S}, \forall \tau_{k-1}\left(t_{k}^{-}\right) \in[\underline{\tau}, \bar{\tau}]$. Hence, condition (30) is indeed verified and we conclude that

$$
x\left(t_{1}\right)^{\prime} P(0) x\left(t_{1}\right)<x\left(t_{1}^{-}\right) P\left(\tau_{0}\left(t_{1}^{-}\right)\right) x\left(t_{1}^{-}\right) \leq 1
$$

Thus, repeating the reasoning, it follows that $x\left(t_{k}^{-}\right) \in$ $\mathcal{S} \forall k \in \mathbb{N}$, and the proof is concluded.

\section{OPTIMIZATION PROBLEM}

For a given upper and lower bound on the sampling period, a region of initial conditions for the closed loop system with guaranteed asymptotic stability can be computed, i.e., provided that $x(0) \in \mathcal{E}(P(0))$, Theorem 2 guarantees that the trajectory of $x$ converges asymptotically to the origin.

The idea is to maximize region $\mathcal{E}(P(0))$ considering some size criterion. The maximization of the minor axis of the ellipsoid is adopted here as a criterion, leading to the following optimization problem:

$$
\begin{aligned}
& \min _{P_{1}, P_{2}, G_{j}, N, \varepsilon} \varepsilon \\
& \text { subject to: } \\
& (17),(18),(19),(20), \\
& (21),(22),(23),(24) \\
& \text { and } P_{1}-\varepsilon I<0
\end{aligned}
$$

The last constraint in problem (33) ensures that the maximal eigenvalue of $P_{1}$ is smaller than $\varepsilon$. Hence, the 
minimization of $\varepsilon$ corresponds to the maximization of the minor axis of the ellipsoid $\mathcal{E}(P(0))$, since $P(0)=P_{1}$. Problem (33) is not convex since the product between $G_{j}$ and $T$, i.e. the constraints are not LMIs. Thus, a way of overcoming this problem and obtaining LMI constraints consists in fixing $T$. This limitation will be worked around in a future study, but if we choose to deal with a SISO problem, where $m=1$, this is not a serious limitation because $T$ is a scalar. Thus, the optimal value of (33) can be easily obtained by solving LMI problems over a grid in $T$.

Remark: As in Fiacchini and Gomes da Silva Jr. (2018), it is important to remark that the region $\mathcal{E}(P(0))$ is defined for $x(t)=\left[x_{p}(t)^{\prime} u(t)^{\prime}\right]^{\prime}$. Observe that the variables $x_{p}(0)^{\prime}$ and $u(0)^{\prime}$ are related by the following:

$$
u(0)=\operatorname{sat}\left(K x_{p}(0)\right)=\operatorname{sat}\left(K x_{0}\right)
$$

Therefore, a guaranteed set of plant initial states, i.e. the set in the plant subspace such that $x(0)=\left[x_{p}(0)^{\prime} u(0)^{\prime}\right]^{\prime} \in$ $\mathcal{E}(P(0))$ is actually described by the set

$$
\begin{aligned}
& \mathcal{X}= \\
& \left\{x_{p} \in \mathbb{R}^{n} ;\left[\begin{array}{c}
x_{p} \\
\operatorname{sat}\left(K x_{p}\right)
\end{array}\right]^{\prime} P_{1}\left[\begin{array}{c}
x_{p} \\
\operatorname{sat}\left(K x_{p}\right)
\end{array}\right] \leq 1, \tau \in[0, \bar{\tau})\right\}
\end{aligned}
$$

which defines a piecewise quadratic region in $\mathbb{R}^{n}$.

\section{NUMERICAL EXAMPLE}

Consider the system (10) with the following matrices:

$$
\begin{aligned}
& A=\left[\begin{array}{cc}
-0.3 & 1 \\
1 & -0.3
\end{array}\right] ; B=\left[\begin{array}{l}
0 \\
2
\end{array}\right] ; \\
& K=\left[\begin{array}{ll}
-1.5 & -1
\end{array}\right]
\end{aligned}
$$

We assume that the intersampling times $\delta_{k} \in[0.05,0.1]$. Solving (33) on a grid on $T$, the minimum is obtained for $T=0.1$, leading to matrices:

$$
\begin{gathered}
P_{1}=\left[\begin{array}{lll}
0.5348 & 0.3688 & 0.1225 \\
0.3688 & 0.3915 & 0.1146 \\
0.1225 & 0.1146 & 0.1111
\end{array}\right] \\
P_{2}=\left[\begin{array}{lll}
-0.4604 & -0.7215 & -0.8192 \\
-0.7215 & -0.5089 & -0.8726 \\
-0.8192 & -0.8726 & -0.4589
\end{array}\right]
\end{gathered}
$$

The region of stability $\mathcal{X}$ is shown in Figure 1 . As a comparison, in Figure 1 is also plotted the domain obtained with the conditions proposed by Fiacchini and Gomes da Silva Jr. (2018). It can be observed that for both estimates, since $\mathcal{X}$ is defined from a piecewise quadratic function, the region estimated is not necessarily convex. One advantage of the method of this study, however, is that the optimization problem is far simpler in terms of its conditions. Finally, Figure 2 contains several trajectories obtained from initial conditions at the boundary of region $\mathcal{X}$, while considering $\delta_{k}$ randomly chosen in the interval $\delta_{k} \in[0.05,0.1]$, showing the expected convergence of all trajectories.



Figure 1. Region $\mathcal{X}$ obtained with the proposed method (red) and region obtained with the algorithm of Fiacchini and Gomes da Silva Jr. (blue).

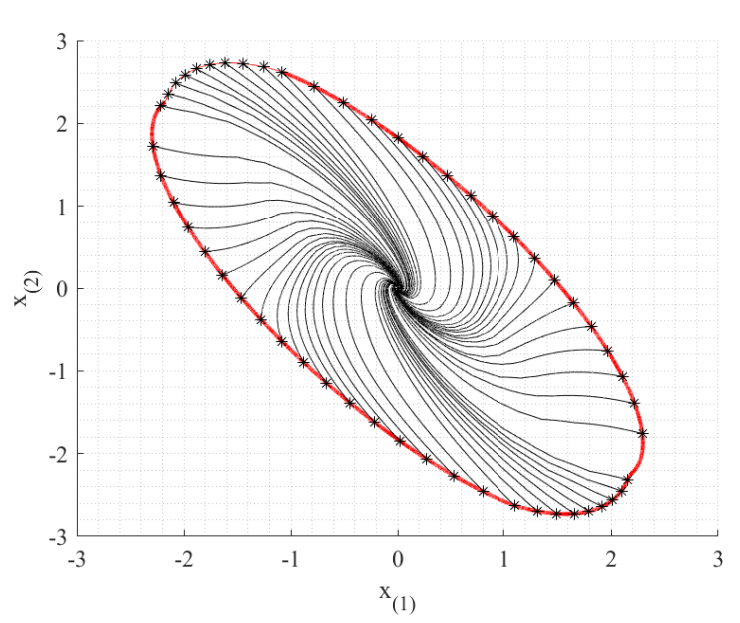

Figure 2. Trajectories starting at the boundary $\mathcal{X}$, with random sampling intervals

\section{CONCLUSION}

The new approach for stability analysis proposed by this paper has been tested and also compared with another current method, showing positive results. This study is actually an early stage of an ongoing work considering more generic clock dependent Lyapunov functions, namely depending polynomially on the clock variable. This will require for instance the use of SOS programming (Prajna et al., 2004) to solve the stability conditions. A study in this sense has been already conducted by Briat (2016) without the saturation effect, which is not straightforward to consider. With this generalization, it is expected to obtain less conservative estimates of the region of attraction.

\section{REFERENCES}

Aström, J.J. and Wittenmark, B. (1984). Computer Controlled Systems: Theory and design. Information and System Sciences Series. Prentice-Hall.

Briat, C. (2016). Theoretical and numerical comparisons of looped functionals and clock-dependent lyapunov functions - the case of periodic and pseudo-periodic systems 
with impulses. International Journal of Robust and Nonlinear Control, 26, 2232-2255.

Chen, T. and Francis, B. (1994). Optimal sampled-data control systems.

Dai, D., Hu, T., Teel, A., , and Zaccarian, L. (2009). Output feedback design for saturated linear plants using deadzone loops. Automatica, 45(12), 2917-2924.

Fiacchini, M. and Gomes da Silva Jr., J.M. (2018). Stability of sampled-data control systems under aperiodic sampling and input saturation. In 57th IEEE Conference on Decision and Control, 1286-1293. Miami Beach, USA.

Finsler, P. (1937). Über das vorkommen definiter und semidefiniter formen in scharen quadratischer formen. 9, 188-192.

Geromel, J.C. and Souza, M. (2005). On an LMI approach to optimal sampled-data state feedback control design. International Journal of Control.

Gomes da Silva Jr., J.M., Queinnec, I., Seuret, A., and Tarbouriech, S. (2016). Regional stability analysis of discrete-time dynamic output feedback under aperiodic sampling and input saturation. IEEE Transactions on Automatic Control, 61, 4176-4182.

Hespanha, J.P., Naghshtabrizi, P., and Y.Xu (2007). A survey of recent results in networked control systems. Proceedings of the IEEE, 95(1), 138-162.

Hetel, L., Fiter, C., Omran, H., Seuret, A., Fridman, E., Richard, J., and Niculescu, S. (2016). Recent developments on the stability of systems with aperiodic sampling: An overview. Automatica, 76, 309-335.

$\mathrm{Hu}, \mathrm{T}$. and Lin, Z. (2001). Control systems with actuator saturation: analysis and design. Boston: Birkhauser.

P. Antsakils, J.B. (2007). A special issue on technology of networked control systems. 95(1), 5-8.

Prajna, S., Papachristodoulou, A., Valmorbida, J., Anderson, J., Seiler, P., and Parrilo, P. (2004). SOSTOOLS: sum of squares optimization toolbox for matlab.

Seuret, A. and Gomes da Silva Jr., J.M. (2012). Taking into account period variations and actuator saturation in sampled-data systems. Systems \& Control Letters, 61, 1286-1293.

Tarbouriech, S., Garcia, G., Gomes da Silva Jr., J.M., and Queinnec, I. (2011). Stability and Stabilization of Linear Systems with Saturating Actuators. Springer. 